\title{
Auditing of Nursing Care for Hemodialysis Patients at Ma,an Governmental Hospitals- Jordan
}

\author{
Khaldoon Aied Alnawafleh ${ }^{1}$, Zienab Abd. El-Latif Mohammad', Hani Abbas Al-Nwafleh ${ }^{3}$. \\ 1. Lecture of Medical Surgical, Faculty of Nursing \&Health Science, AL-Hussein Bin Talal University, Jordan. \\ 2. Prof of Medical Surgical Nursing, Faculty of Nursing, Assiut University, Egypt. \\ 3. Prof. of Community Health Nursing, Faculty of Nursing \&Health Science, AL-Hussein Bin Talal University, \\ Jordan.
}

\begin{abstract}
Nursing auditing is used to evaluate the nursing care given to achieve deserved and feasible quality of nursing care. Aims of this study were to assess current nursing performance for patients undergoing hemodialysis, Develop nursing care standards and identify the gap between the current nursing management and nursing care standards for those patients. Research design: Exploratory research design was used . Setting: The study was conducted in dialysis unit at Ma'an governmental hospitals- Jordan Subjects: The sample in this study is composed of all available nurses (40 nurses) who are providing direct care to patients in hemodialysis units at previously mentioned hospital. Tools of Data Collection: Self-Administered Interview Questionnaire sheet, an observational check, developed Nursing Care Standards for Patients undergoing haemodialysis, and Nursing Care Standards checklist. Results: Around half of nurses their knowledge score ranged between good and fair regarding renal failure and dialysis. Only $25 \%$ of nurses done correctly in nursing standers 4 about patient educated before discharge. Conclusion: about quarter of nurses perform after dialysis steps incorrectly. There was no statistical significant difference between routine care and standers nursing care for patient undergoing hemodialysis. Recommendations: Written standards of care against which to evaluate nursing care. Training for auditors should include a group discussion to see how the group rates the care received using the notes of a patient.
\end{abstract}

\section{Key words: Auditing, Nursing Care \& Hemodialysis Patients.}

\begin{abstract}
Introduction
Adequate kidney function is essential to the maintenance of a healthy body. If a person has complete kidney failure and treatment is not provided, death is inevitable (Lewis et al., 2014).

Prevalence of ESRD patients in Jordan (2015); The total numbers of patients treated and registered in the Jordan Renal Registry by the end of 2015 was (4935) patients; of them 4690 were Jordanians (95\%) and 245 were non Jordanians (5\%) (Annual Report, Jordan, 2015).

The International comparison of prevalence rate of Hemodialysis patients Per Million Population (PMP) in Jordan and other countries in 2015. The overall Prevalence Per Million Populations in Jordan in 2015 is $(709.1 / 1,000,000)$ compared with other countries (Annual Report, Jordan, 2015).

Nursing services are necessary for every patient seeking care of any type, including health promotion, diagnosis and treatment. With the changing trends in the health care delivery, the role of the nurse manager is becoming largely devoted to the holistic care of patient which can only achieved through the careful appraisal of the services in order to make further reforms (Amanda, 2014).

Audit is a systematic and critical examination to examine or verify. Nursing audit; it is the assessment of the quality of nursing care and uses a record as an
\end{abstract}

aid in evaluating the quality of patient care (Viana et al., 2016).

Nursing audit, is a review of the patient record designed to identify, examine, or verify the performance of certain specified aspects of nursing care by using established criteria (Mosby's Medical Dictionary, 2009).

Nursing audit is the process of collecting information from nursing reports and other documented evidence about patient care and assessing the quality of care by the use of quality assurance programmes (Johnson, Jefferies, \& Langdon, 2010).

A concurrent nursing audit is performed during ongoing nursing care. A retrospective nursing audit is performed after discharge from the care facility, using the patient's record. To assure effective patient care, comprehensive audit tools are required (Johnston, 2010).

A profession concerns for the quality of its service constitutes the heart of its responsibility to the public. An audit helps to ensure that the quality of nursing care desired and feasible is achieved (Jamtvedt, 2010).

The function of nurse auditor is expanding in the job market, mainly regarding the analysis of the hospital accounts, which consists in verifying the compatibility between what was consumed, what is 
being charged and the procedures actually performed (Hess, 2010).

\section{Significance of the study}

Through clinical researchers experience it has been observed that; patients undergoing dialysis need detailed evaluation of many components of health care throughout relevant audit care standards. Therefore, this study will be the first in this geographical location which could be helpful for health professionals especially nurses in planning and implementing care for such group of patients.

\begin{abstract}
Aim of the study
Aims of this study were covering the following to : Assess current nursing performance for patients undergoing hemodialysis, develop nursing care standards for patients undergoing hemodialysis and to Identify the gap between the current nursing management and nursing care standards for patients undergoing hemodialysis.
\end{abstract}

\section{Subjects \& Method \\ Research design:}

Exploratory research design has been utilized used to achieve the aim of the study.

\section{Technical Design}

\section{Setting of the study}

The study was conducted in dialysis unit at Ma'an governmental hospitals- Jordan (Ma'an governmental hospitals and Ministry Queen Rania Al Abdullah Hospital)

\section{Study Subjects}

The sample in this study was composed of all available nurses (40 nurses) who were providing direct care to patients in hemodialysis units at previously mentioned hospitals.

\section{Tools of Data Collection}

Three tools of the study were utilized for data collection and developed by the researcher, it included the following:

Tool I: Nursing performance Questionnaire sheet

The questionnaire was constructed by the researcher based on review of literature to asses Nurses knowledge and practice about hemodialysis. It includes the following parts:

Part 1: Personal and demographic data of the nurses as age, level of education and years of experience .....etc.

Part 2: Self-Administered Interview Questionnaire sheet: it was used to asses nurse's knowledge about nursing care of patients undergoing hemodialysis.it include multiple of choice questions about structure and function of kidney, types, treatment, management of renal failure, definition. It also include list questions about fistula and dialysis management (pre, during and post dialysis scission).

Scoring system

The total score for all items was 80 marks; those who obtained less than $50 \%$ were considered having poor level of knowledge. While those who obtained from (50\%-70\%) fair, but above $70 \%$ were considered having good level of knowledge.

Part 3: Observation checklist sheet for patients undergoing hemodialysis Annex (B)

An observational check list was used to assess practice of nurses during hemodialysis session, (initiation of dialysis, nursing intervention with common problems during hemodialysis session and at termination of dialysis).

Scoring system:

The total score for all items was 130 marks .each correct done was given one score. Those who abstained less than $50 \%$ were considered having poor level of performance. While those who obtained from (50\%-70\%) fair, but above $70 \%$ were considered having good level of performance.

Tool II: The developed Nursing Care Standards for Patients undergoing Haemodialysis.

This tool was developed by the researcher after reviewing current national and international literature. It includes nursing care standards based on Control Disease Centre (CDC) (Sidawy et al., 2002 \& O'Grady et al., 2002) and Healthy Care Accreditation Council (HCAC) at Jordan to compare nursing practice with the standard guideline's recommendations. It included a teaching booklet which was written in Arabic using simple language with illustrations and it was concerning knowledge about:

- Brief illustrations of the anatomy of the kidney.

- Definition, causes, and clinical manifestations of end sage of renal failure.

- Diagnostic studies and treatment of renal failure.

- Haemodialysis: definition, indications, advantages, disadvantages and fistula types.

Developed Nursing care standard:

Standard 1: Ensure the dialysis unit is available with a safe environment to receive the patient.

Standard 2: Ensure that infection control methods are followed in each nursing procedure.

Standard 3: Ensure the provision of nursing care for all dialysis patients (before, during and after dialysis cession).

Standard 4: Ensure that the patient receives instructions and instructions before leaving the hospital. 
Tool III: Checklists sheet for nurse's performance about dialysis

(Nursing Care Standards)

This tool was developed by the researcher to achieve the aim of study (3) to identify the gap between the current nursing performance and nursing care standards for patients undergoing hemodialysis. It cover the four standard, standard (1) was covered items about ensure that hemodialysis room environment is safe to receive the patients. Standards (2) which covered items about ensure that infection control is properly followed in hemodialysis unit. Standards (3): Ensure that nursing care is provided for Patients undergoing Hemodialysis. This covered items about (Nursing care before, during and after hemodialysis. Finally standards (3): which included items about ensure that patient is properly educated before discharge. Each stander had sub-items and each sub items were observed by the researcher and cheeked done, not done or not applicable.

\section{Methods}

\section{Operational design}

Tool testing and pilot study

Pilot study: A pilot study was conducted on $10 \%$ of the study sample (4 nurses) in a selected setting to evaluate the applicability \& clarity of the tools. According to this pilot study, the required modifications were made. Those patients who were involved in the pilot study were not included in the study.

Content validity: It was established by a jury of five experts (2 professors in medical surgical nursing and 3 professors in urology specialty) who was reviewed the instruments for clarity, relevance comprehensiveness, understanding, applicability, and easiness for administration.

Reliability: was estimated by Alpha Cronbach's test result was $\mathrm{R}=0.68$

\section{Technique for data collection}

A structured self-administered questionnaire to fill out the questionnaire sheet (tool I part a, b).Also observational technique was utilized to fill out the checklist for practice of nurses (tool I, part 3). And Checklists sheet to assess nurse's performance about dialysis: (Nursing Care Standards) Tool III.

\section{Administrative design}

Permission to carry out the study was obtained from Dean faculty of nursing, ministry of health and the responsible hospital authorities of $\mathrm{Ma}$ 'an Governmental hospital.

\section{Ethical considerations}

1. Research proposal was approved from Ethical Committee in the faculty of nursing.

2. There is no risk for study subject during application of research.
3. The study was following common ethical principles in clinical research.

4. Written consent was obtained from nurses that who are willing to participate in study, after explaining the nature and purpose the study.

5. Study subjects had the right to refuse to participate and or withdraw from the study without any rational at any time.

6. Confidentiality and anonymity of study subject was assured.

\section{Procedure}

The study was carried out on 3 phases

Phase (1): preparatory phase:

In this phase, the researcher designed and tested the developed nursing care standard after extensive literature review ( nursing and medical textbooks, journal, internet resources, etc.) about dialysis and assessment of nurses, knowledge and practice in this regard ( preparation of data collection tools).

\section{Phase (2): planning phase}

Based on finding of the exploratory phase, the developing nursing care standard was developed based on Control Disease Centre (CDC) (Sidawy et al., $2002 \&$ O'Grady et al., 2002) and Healthy Care Accreditation Council (HCAC) at Jordan to compare nursing practice with the standard guideline's recommendations. after extensive literature review considering nursing needs and their level of understanding.

Phase (3): Implementing Phase: The third phase (implementation phase), in which an official permission to proceed with the proposed study was obtained from the head of dialysis units.

At initial interview the researcher introduce himself to initiate communication, explain the nature and purpose of the study and ask the nurses to fill out the questionnaire sheet (tool I), to assess nurses knowledge. The researcher fills out observation checklist to assess nurse's performance.

\section{Statistical analysis}

An appropriate statistical methods \&tests will be used for analysis of the results. Data collected will be analyzed \& tabulated using frequency percentage \& the suitable tests. 


\section{Results}

Table (1): Frequency distribution of demographic characteristic among nurses participant $n=32$

\begin{tabular}{|l|c|c|}
\hline \multicolumn{1}{|c|}{ Variables } & N & \% \\
\hline Age group & & 0 \\
$18 \geq 20$ years old & 0 & 59.4 \\
$20 \geq 30$ years old & 19 & 28.1 \\
$30 \geq 40$ & 9 & 12.5 \\
40 years and above & 4 & \\
\hline Sex & 22 & 68.8 \\
Male & 10 & 31.3 \\
Female & 9 & \\
\hline Marital status & 22 & 281 \\
Single & 1 & 68.8 \\
Married & 0 & 3.1 \\
Divorced & & 0 \\
Widow or widower & --- & \\
\hline Level of education & 4 & --- \\
School of Nursing & 28 & 87.5 \\
Nursing Institute & & \\
Nursing College & 17 & 53.1 \\
\hline Years of experience & 15 & 46.9 \\
Less than 5 years & & \\
More than 5 years & 11 & 34.4 \\
\hline Have you received specialized training in dialysis! & 21 & 65.6 \\
\hline Yes & 32 & 100.0 \\
\hline No & & \\
\hline Total & & \\
\hline
\end{tabular}

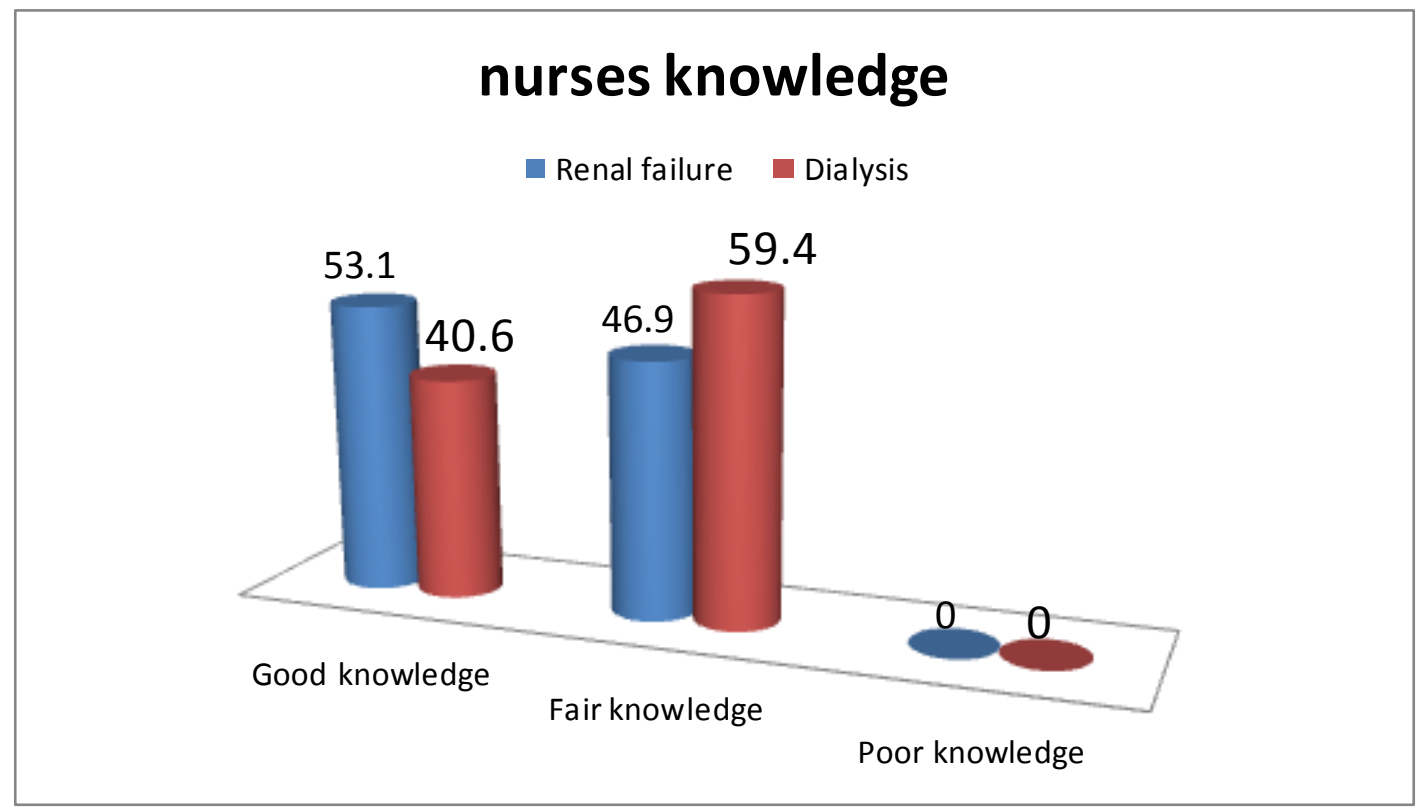

Fig. (1): distribution for level of nurse's knowledge about renal failure and dialysis $n=32$ 


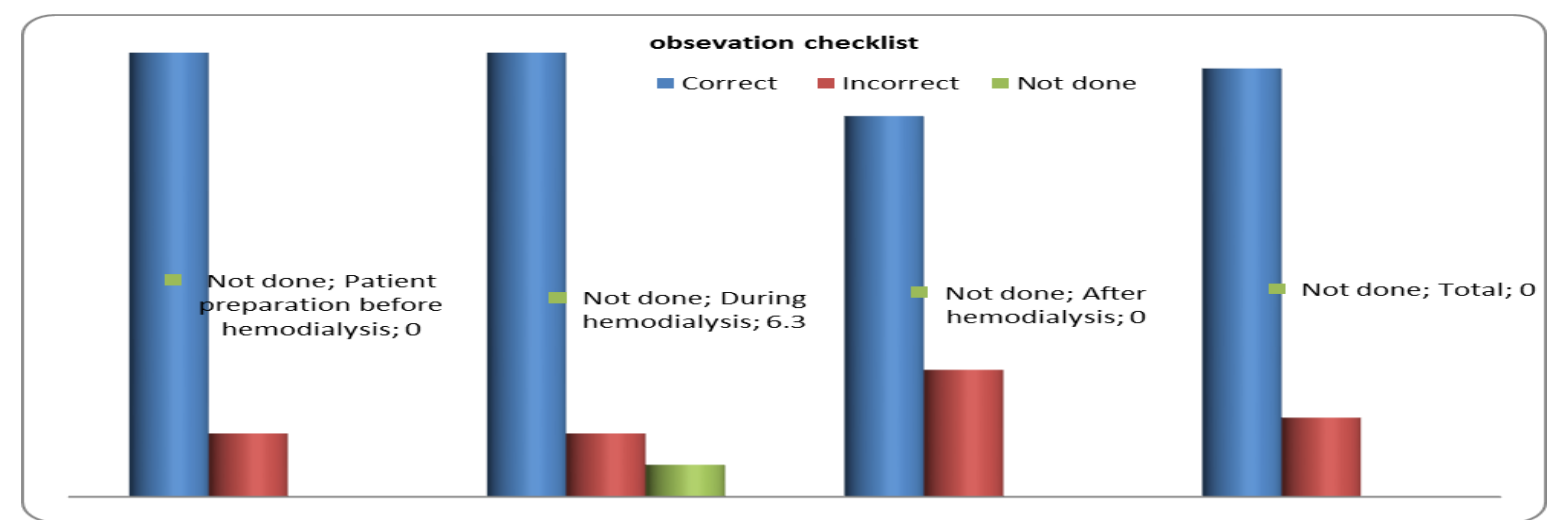

Fig.(2): Frequency distribution for nursing care of total observation checklist for patients undergoing hemodialysis dialysis $\mathbf{n}=32$ dialysis and level of nurses' knowledge $n=32$

Table (2): Relation between routine nursing care of observation checklist for patients undergoing hemodialysis

\begin{tabular}{|c|c|c|c|c|c|c|c|}
\hline \multirow{3}{*}{$\begin{array}{r}\text { Items } \\
\text { Good knowledge }\end{array}$} & \multicolumn{2}{|c|}{ Correct } & \multicolumn{2}{|c|}{ incorrect } & \multirow{2}{*}{\multicolumn{2}{|c|}{ Total }} & \multirow{2}{*}{ p.va } \\
\hline & $\mathbf{N}$ & $\%$ & $\mathbf{N}$ & $\%$ & & & \\
\hline & 14 & 43.8 & 1 & 3.1 & 15 & 45.6 & \multirow{3}{*}{$0.208^{\mathrm{ns}}$} \\
\hline Fair knowledge & 13 & 40.6 & 4 & 12.5 & 17 & 54.4 & \\
\hline Poor knowledge & 0 & 0 & 0 & 0 & 0 & 0 & \\
\hline
\end{tabular}

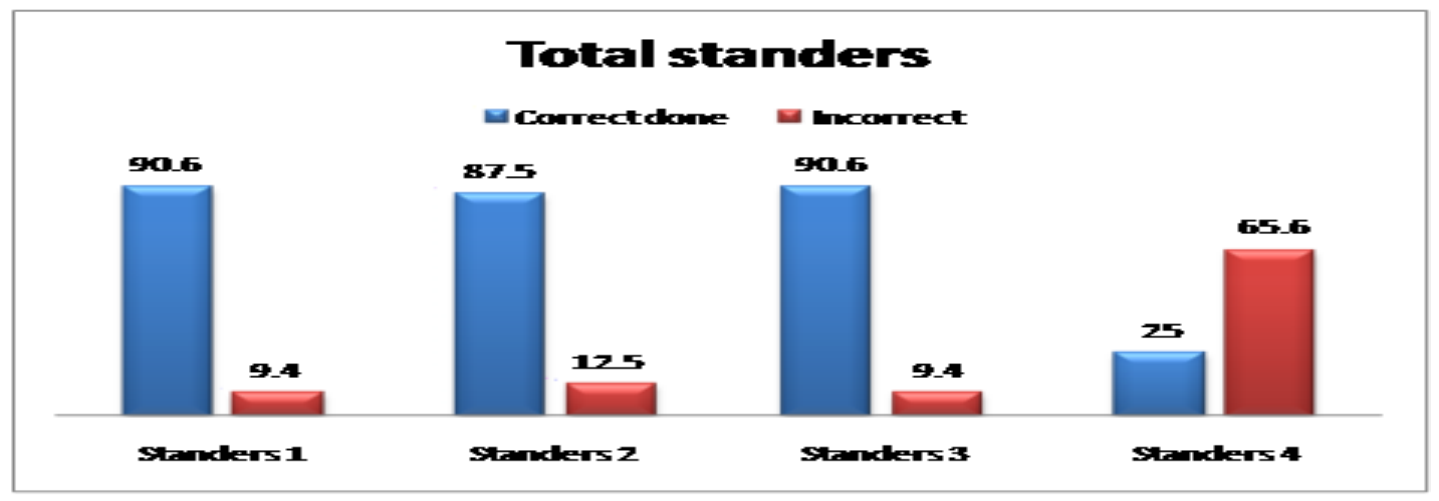

Fig. (3): Total standers nursing care is provided for Patients undergoing Hemodialysis. $n=32$

Table (1): Shows that more than half of nurses were aged between 21 to less than 30 years old, male, married, have less than five years of experience and haven't received specialized training courses in dialysis. Regarding the level of education; the majority of studied sample had a college education.

Fig. (1) : Represents that the level nurse's knowledge about dialysis more than half of nurses have a good level of knowledge about renal failure, and have a fair level of knowledge regarding dialysis. Fig (2): Shows that the majority of nurses performing all steps of observation checklist for patients before, during, and after hemodialysis procedure correctly except one quarter of nurses perform after dialysis steps incorrectly.
Table (2): Clarify the relation between routine observation checklist for patients undergoing hemodialysis dialysis and level of nurses' knowledge. It shows that there was no statistical significant difference between routine observation checklist for patients undergoing hemodialysis dialysis and level of nurses' knowledge.

Fig (3): This fig. shows that the majority of nurses perform the steps of first, second and the third standards correctly, while more than half of nurses perform the steps of the fourth standard incorrectly. 


\section{Discussion}

Nursing audit is a management tool capable of assisting in the improvement of care quality and efficiency of hospital charges and may be performed concurrently, in other words, while the patient receives care. A clinical audit can identify activities that should or should not be performed in clinical settings. It not only improves care quality but also helps nurses to acquire and use the knowledge, skills, and attitudes necessary for creating a meaningful, productive, and satisfying working environment (Poortaghi, et al., 2015).

\section{I: Nurses and demographic data}

The results of the present study revealed that more than half of nurses were aged between twenty to less than thirty years old, male, married, have less than five years of experience and haven't received specialized training courses in dialysis. Regarding the level of education; the majority of studied sample had a nursing college education.

Ahmed, (2011), was in the same line with the present study results except concerning the gender of nurses, educational level, and their years of experience as reported that "the majority of the nurses their ages ranged from twenty to thirty years, married, females, and have diploma of nursing. The majority of them have no in-service training courses related to dialysis. The majority of them, their experiences range from five to more than ten years".

Also, this study agree with Mustafa, (2010), who reported that the gender of nurses and their educational level who conducted a study in kidney dialysis unit of El Minia University Hospital entitled 'Measuring nurses' compliance with patients' safety measures during hemodialysis" which revealed that the majority of nurses were married, females, their age ranged from twenty to twenty nine years, have a diploma qualification, years of experiences in hemodialysis less than five years and haven't inservice training courses related to dialysis.

Al- mawsheki, et al., (2016), who reported that " the majority of study sample were females, and more than half of studied nurses their ages ranged between twenty to less than thirty years. More than half of studied nurses had diploma of nursing. Half of studied nurses had experience from one year to less than ten years, and more than one third of studied nurses were attended training courses about patients care in Hemodialysis Unit".

While this is in disagreement with El-Moghazy, (2013) that performed a study titled Nurses Knowledge and Practice Regarding Intradialytic Complications for Hemodialysis Patient stated that "more than half of nurses aged more than thirty years".
II- Nurse's knowledge about nursing care of patients undergoing hemodialysis:

Education needs are considerable in dialysis facilities and include orientation and initial competency testing of all staff, as well as ongoing education and retesting. The development and provision of a patient care technician training program must include the elements of patient care, understanding end stage renal disease and its related conditions, machine technology, water treatment, infection control, patient education concepts Yoder, et al., (2013).

Marquis and Huston, (2009): told that education and training are two components of staff development that occur after an employees' indoctrination (which refers to planned, guided adjustment of employee to the organization and work environment). The staffs' knowledge level and capabilities are a major factor in determining the number of staff required to carry out unit goals. The better trained and more competent the staff, the fewer staff required, which in turn saves the organization money and rise reproductively.

The present study revealed that more than half of nurses have a good level of knowledge about renal failure, and have a fair level of knowledge regarding dialysis. (Antony, et al., 2016), was in the same line with the present study as mentioned that "Comparing the level of knowledge between staff nurses and nursing students, staff nurses having adequate level of knowledge than Nursing students regarding care of patients undergoing hemodialysis".

This is while in disagreement with (Hassona, 2011), who told that "most of nurses had unsatisfactory total knowledge regarding hemodialysis. It might be due to many factors including new graduation, and shortage of attending training courses".

Also, (Ahmed, 2011), was disagreeing with the study results as reported that" Data collected before the designed nursing protocol implementation (pre-test) showed unsatisfactory level of knowledge about renal failure, hemodialysis and care of patients undergoing hemodialysis, which reflects the lack in their scientific preparation".

III- Nurse's skills about nursing care of patients undergoing hemodialysis:

The majority of nurses in the current study perform correctly all basic steps before hemodialysis procedure except two steps (Draw blood sample, review blood chemistries, Dispose gloves and wash hands). Regarding Cannulation of arterio-venous fistula; the majority of nurses perform all steps correctly except Infuse flush solution, and reclamp needle it was not done. The majority of nurses performing all steps of accessing a vascular catheter correctly. Finally, the majority of nurses performing most steps of observation checklist for patients 
before, during, and after hemodialysis procedure correctly

Ali, (2013), who performed a study to assess nurses' knowledge and practice provided to the patients undergoing hemodialysis at Cairo University Hospital was in the same line and stated that " nearly two thirds of nurses had a satisfactory level of practice regarding care during hemodialysis".

The present study showed a significant

While, Goodkin, et al., (2010), was disagreeing with these results as stated that "The current study results revealed that the studied nurses had unsatisfactory practice regarding care for patients during hemodialysis. It might be due to the lack of nurses' application of knowledge especially regarding to nursing interventions with common complications that occur and misunderstanding of their roles as there is no job description or definition of responsibilities in the hemodialysis unit and due to carelessness of nurses". Also, these findings in a disagreement with Hassona, (2011), who stated that all nurses had unsatisfactory knowledge and practice regarding care during hemodialysis.

This is compatible with findings of a study in Sudan by Abdelsatir, (2013), who found that "nurses' performance among nurses of hemodialysis was moderate such as hand washing, assessing patients when there were nausea, dizziness, muscle cramps, checking vital signs, withdraw blood samples for testing serum electrolytes and waste products and patient education".

The current study revealed that "there was no any statistical significant difference between routine observation checklist for nurses (nurses' practice) undergoing hemodialysis and level of nurses' knowledge". Al- mawsheki, et al., (2016), was in the same line with the current results as mentioned that" There is no statistical significant difference between nurses' knowledge and their practice". Also, Ahmed, (2011), reported that" The results in the present study showed no significant difference between nurses' knowledge and their practice observed during the pre-test. This may be attributed to insufficient courses related to hemodialysis included in their undergraduate curriculum of nursing education and also there are no available Arabic sources for updating and continuing their education".

IV- Nurse's care standards for patients undergoing hemodialysis

The majority of nurses perform correctly all steps of the first standard which is ensures that hemodialysis room environment is safe to receive the patients which are (Prepare patient room, Prepare all equipment, preventing patient falls, Reporting accidents and errors / Fire safety, and
Maintaining safe environment and administration of medications).

This will in the same line with (Blankschaen, et al., 2016) who mentioned that" Physical environment requirements of the dialysis unit safety, equipment maintenance, the patient care setting, emergency preparedness, and fire safety. This includes the ensurance of proper maintenance and repair of such items as the dialysis equipment, operating systems, water treatment systems, and physical building and grounds. In a health care space such as a dialysis facility, patients usually occupy nearly seventy percent of the space, with other services using the remaining thirty percent.

Regarding standers 2, the majority of nurses perform incorrectly steps of the second standard which is ensure that infection control is properly followed in hemodialysis unit regarding Principle of aseptic technique in all procedure which are (basics of aseptic technique, Identify and supervise methods of disinfecting, Using universal precaution as hand washing and Applying sterile gloves).

(Krishnan, et al., 2014); clarify that "Infection control is the responsibility of the medical director, and a detailed discussion is beyond the scope of this article. Infection control issues are often cited by state surveyors. All facility staff should be held accountable for infection control. Hand washing is essential for infection control in a hemodialysis unit. Gloves should be worn prior to patient contact or equipment manipulation. Hands should be washed before and after use of gloves. Gloves should be changed when soiled or when moving from one patient chair to another, as well as any time that access manipulation or intravenous medication administration occurs. The facility must be designed with enough sinks to facilitate hand washing, with separate utility sinks for cleaning equipment. Staff should wear personal protective equipment, which includes designated garments with sleeves appropriate to the anticipated potential exposure. The most robust protection should be used during high risk components of the dialysis procedure, such as treatment initiation and termination. Any items that are in the patient's dialysis station could become contaminated and should be handled with caution and cleaned after the treatment (ie, a patient's blanket).

Also, (Allegranzi, et al., 2011), mentioned that "It is universally recognized that the consistent and rigorous implementation of infection control measures reduces considerably the incidence rates of nosocomial infections. Interventions to improve adherence to infection control policies could reduce the prevalence of these infections, allowing more resources to be allocated to other areas of healthcare delivery". 
(Al Qahtani \& Almetrek, 2016), was disagreeing with the study results as reported that "Nurses enrolled in this study showed an overall satisfactory level of practice of infection control procedures reflected by their overall scores of $92.11 \%$.

Regarding standers 3, the majority of nurses perform correctly all steps of the third standard which is ensure that nursing care is provided for Patients undergoing Hemodialysis which are part one includes (basic steps of Nursing care before hemodialysis, Measuring patient's vital signs, and Measuring patient's PH and electrolytes ). Also, Part two which includes Nursing Care during Hemodialysis, and Part three which includes Nursing Care after Termination of the Dialysis Session steps were performed correctly by the majority of nurses.

(Safadi, et al., 2010), was disagreeing with the present study findings as reported that "Nurses showed high practice level regarding regular investigations from patients and types of routine investigations, On the other hand, participants showed moderate level of practice, in which their skills was high regarding measurement of vital signs and weight measuring before dialysis, steps of preparing a hemodialysis machine and meet patients needs during dialysis. But they showed less skills regarding proper use of infection control methods, measuring blood pressure during dialysis and method of counseling".

Concerning standard 4 , the majority of nurses perform incorrectly steps of the fourth standard which is Ensure that patient is properly educated before discharge which are consisting of (Protect the catheter, Activity progression, Healthy Diet) while, nearly half of nurses incorrectly perform the steps of managing the side effects of hemodialysis. Also, nearly half of them didn't know about the common Medications for dialysis patients.

Nurses often have more interaction with patients than any other healthcare professional. Therefore, they are in an optimal position to provide ongoing patient education, support and encouragement, and thus promote self-care efficacy, and overall quality of life (Wells, 2011). Continuous education of nurses is vital in supporting the nurses' unique role as both a patient educator and a patient advocate. Although patient education has a positive effect on knowledge as well as adherence to treatment, it is meager on its own. Combining education with goal setting and reinforcement, and providing patients with additional support can have more desirable outcomes (Pessoa \& Linhares, 2014), Therefore, nurses should not only educate their patients but find ways to motivate them to commit to their care.

(Painter et al., 2011), emphasized the dialysis patient' education on the bio- physiological and functional aspects of managing the treatments. The content of education should include information about kidney function, the disease, and the different treatment options as well as forms of dialysis. In addition, detailed instructions for the care of vascular access sites, education on fluid restrictions, and medication management guidance are required.

\section{Conclusion}

Based on the results of the present study, it can be concluded that:

More than half of nurses have a good level of knowledge about renal failure, and have a fair level of knowledge regarding dialysis. The majority of nurses performing all steps of observation checklist for patients before, during, and after hemodialysis procedure correctly except one quarter of nurses perform after dialysis steps incorrectly. There was no statistical significant difference between routine care and standers nursing care for patient undergoing hemodialysis.

\section{Recommendations}

Based on results of the present study the following can be recommended:

- ESRD care expands and the number of CKD patients' increases, the focus will need to shift from routine care to nursing standard care.

- Understand available audit tools to assure that nursing care plans are monitoring in the most effective way.

- Training for auditors should include a group discussion to see how the group rates the care received using the notes of a patient

- Written standards of care against which to evaluate nursing care.

\section{References}

1. Abdelsatir S., (2013): Evaluation of Nurses Awareness and Practice of Hemodialysis Access Care in Khartoum State, Sudan. Arab Journal of Nephrology and Transplantation. Vol. 6(2):pp.119-21.

2. Ahmed G., (2011): Effect of designed nursing protocol on nurse's knowledge and practice regarding Hemodialysis patients, thesis submitted for partial fulfillment of the requirements of the master degree in adult nursing, discussion part, p.84.

3. Al- Mawsheki E., Ibrahim M., \& Taha N., (2016): Nurses' Knowledge and Practice Regarding Care for the Patients during Hemodialysis, Med. J. Cairo Univ., Vol. 84, No. 1, September: 1135-1141, available at: www.medicaljournalofcairouniversity.net 
4. Al Qahtani A., \& Almetrek M., (2016): Knowledge, Attitudes and Practice of Nurses in Renal Dialysis Units Regarding Infection Control in Abha City - Saudi Arabia, The Egyptian Journal of Hospital Medicine (Jan. 2017) Vol. 66, Page 103-114. 103 Received: 18/10/2016 DOI: 10.12816/0034640 Accepted: 23/10/2016.

5. Ali S., (2013): Assessment of Nurses' Knowledge and Practice Provided to the Patients Under Going Hemodialysis at Cairo University Hospital. Unpublished Master Thesis, Department of Medical Surgical Nursing Science. Faculty of Nursing, Cairo University, discussion part, p.94.

6. Allegranzi B., BagheriNejad S., Combescure C., Graafmans W., Attar H., Donaldson L., (2011): Burden of endemic health-care associated infection in developing countries: systematic review and meta-analysis. Lancet, 377(9761):228

7. Amanda, (2014): Nursing audit- definition, history, purposes, methods, characteristics, process, part 1.

8. Annual Report, Jordan, 2015. Department of Statistics (DOS)

9. Antony L., paramjyothi B., \& Indira S., (2016): A Study to Assess the Knowledge Regarding Care Of Patients Undergoing Hemodialysis among Staff Nurses and Nursing Students in Narayana Medical College Hospital, Nellore. Imperial Journal of Interdisciplinary Research (IJIR) Vol-2, Issue-6, Page 623. ISSN: 2454-1362, http://www.onlinejournal.in Imperial Journal of Interdisciplinary Research (IJIR)

10. Bakey S., (2014): Evaluation of Nurses' Practices throughout Hemodialysis Treatment for Patients in hemodialysis unit at Baghdad teaching hospitals. Kufa Journal for Nursing Sciences, vol. 2: p.2.

11. Blankschaen S., Saha M., \& Sharmeela S., (2016): Core Curriculum in Nephrology Management of the Hemodialysis Unit: American Journal of Kidney Disease. Vol. 68(2):pp. 316-327

12. El-Moghazy G., (2013): Nurses Knowledge and Practice Regarding Intradialytic Complications for Hemodialysis Patient. Journal of American Science, vol. 9: p. 11.

13. Goodkin, K., Pisoni, R., Locatelli, F., Port, F., \& Saran, R., (2010): Hemodialysis vascular access training and practices are keys to improved access outcomes. American Journal of Kidney Disease. Vol. 56: pp.1032-1042
14. Hassona F., (2011): Evaluation of an Educational Program: A Report from the Hemodialysis Unit in Zagazig University Hospitals, Egypt. Nephrology Nursing Journal, vol.39: p.53.

15. Jamtvedt G., (2010): Audit and feedback: effects-on professional practice and health care outcomes. J. Quality in health care. PP. 7:27

16. Poortaghi S., Salsali M., Ebadi A., Rahnavard Z., \& Maleki F., (2015): Findings from a Nursing Care Audit Based on the Nursing Process: A Descriptive Study, Nurs Midwifery Stud. 4(3): e30181.

17. Johnson M., Jefferies D., Langdon R., (2010): The Nursing and Midwifery Content Audit Tool (NMCAT): a short nursing documentation audit tool. Nurs Manag. 18(7):832-45.

18. Krishnan M., Brunelli S., Maddux F., (2014): Guiding principles and checklists for population-based quality metrics. Clin J Am Soc Nephrol.vol;9 (6):pp.1124-1131.

19. Lewis S., Dirksen S., Heitkemper M., Bucher L., \& Harding M., (2014): Medical Surgical Nursing, Assessment and Management of clinical problems, 9th (ed), by Mosby, an imprint of Elsevier Inc. Chapter 45. PP. 1046 1063, 1107 -

20. Marquis L., \& Huston J., (2009): Leader ship roles \& management functions in nursing, 6th ed., Lippincott, Hong Kong, p. 371.

21. Mosby's Medical Dictionary, 8th edition. (C) (2009): Elsevier.

22. Mostafa A., (2010): Measuring nurses' compliance with patients 'safety measures during hemodialysis at El Minia Hospitals. Submitted for partial fulfillment of the requirements for the Master Degree in nursing Administration, Faculty of Nursing, El Minia University, discussion part, p.95.

23. Painter, P., Ward, K., \& Nelson, R., (2011): Self-reported physical activity in patients with end stage renal disease. Nephrology Nursing Journal, 38(2), 139-148.

24. Pessoa, N., \& Linhares, F., (2014): Hemodialysis patients with arteriovenous fistula: Knowledge, attitude and practice. Anna Nery School Journal of Nursing, 19(1), 73-79.

25. Safadi R., Jaradeh M., Bandak A., Froelicher E., (2010): Competence assessment of hemodialysis nursing graduates of Jordanian universities. Nursing and Health Sciences. Vol. 12(2):pp.147-54.

26. Viana C., Todendi L., Lazzari D., Garcia C., \& Souto G., (2016): Implementation of Concurrent Nursing Audit: An Experience 
Report. Texto contextoenferm. vol.25 no.1 Florianópolis 2016 Epub Apr 01

27. Wells, J., (2011): Hemodialysis knowledge and medical adherence in African Americans diagnosed with end stage renal disease: results of an educational intervention. Nephrology Nursing Journal, 38(2), 155-162.

28. Yoder L., Xin W., Norris K., Yan G., (2013): Patient care staffing levels and facility characteristics in US hemodialysis facilities. Am J Kidney Dis.vol.62(6):pp.1130-1140.

29. Sidawy, A., Gray, R., Besarab, A., Henry, M., Ascher, E., Silva J., \& Rutherford, R., (2002): Recommended standards for reports dealing with arteriovenous hemodialysis accesses. Journal of vascular surgery, 35(3), 603-610

30. O'Grady, N., Alexander, M., Dellinger, E., Gerberding, J., Heard, S., Maki, D., \& Raad, I., (2002): Guidelines for the prevention of intravascular catheter-related infections. Centers for Disease Control and Prevention. MMWR. Recommendations and reports: Morbidity and mortality weekly report. Recommendations and reports, 51(RR-10), 1-29. 